\title{
Autophagy-related genes are potential diagnostic biomarkers for dermatomyositis
}

\author{
Le Wang ${ }^{1}$, Dalang Fang ${ }^{2}$, Yuan Liu ${ }^{1}$ \\ ${ }^{1}$ Department of Rheumatology, Liuzhou People's Hospital, Liuzhou, China; ${ }^{2}$ Department of Breast and Thyroid Surgery, The Affiliated Hospital of \\ Youjiang Medical University for Nationalities, Baise, China \\ Contributions: (I) Conception and design: Y Liu; (II) Administrative support: Y Liu; (III) Provision of study materials or patients: L Wang; (IV) \\ Collection and assembly of data: D Fang; (V) Data analysis and interpretation: D Fang; (VI) Manuscript writing: All authors; (VII) Final approval of \\ manuscript: All authors. \\ Correspondence to: Yuan Liu. Department of Rheumatology, Liuzhou People’s Hospital, Liuzhou, China. Email: liuyuanem@163.com; Dalang \\ Fang. Department of Breast and Thyroid Surgery, The Affiliated Hospital of Youjiang Medical University for Nationalities, Baise, China. \\ Email: fangdalang@stu.gxmu.edu.cn.
}

Background: Dermatomyositis (DM) is an autoimmune disease mainly diagnosed by its symptoms and a physical examination, with only some subtypes of DM showing clear molecular changes. To date, few biomarkers have been identified to assess DM progression. Autophagy-related genes have been significantly correlated with inflammation, several types of autoimmune diseases, and the immune response, but few studies have explored the role of autophagy-related genes in DM. Therefore, this study aimed to investigate the roles of autophagy-related genes in DM.

Methods: We collected three datasets of dermatomyositis-related transcriptome from the Gene Expression Omnibus (GEO) database: GSE1551, GSE46239, and GSE143323 and analyzed the differentially expressed genes (DEGs). We also conducted functional enrichment analyses with Gene Ontology (GO) and Kyoto Encyclopedia of Genes and Genomes (KEGG) pathways. To explore whether the autophagy-related genes were differentially expressed in DM compared with normal samples, we performed an intersection between the DEGs and autophagy-related genes obtained from the Human Autophagy Database (HADb, http:// www.autophagy.lu/). Finally, we used selected autophagy-related genes as biomarkers for diagnosing and analyzing the correlation with immune cell infiltration.

Results: Our results showed that 143 genes were upregulated, and 14 were downregulated in the DM samples compared with healthy samples. The functional enrichment analysis revealed that these DEGs played a significant role in the type I interferon signaling pathway, cytokine activity, chemokine activity, double-stranded RNA binding, and blood microparticles. The intersection results identified CCL2, CDKN1A, FOS, MYC, and TNFSF10 as the primary autophagy-related genes in DM. All showed significantly increased expressions in DM samples compared with healthy samples. We were also curious to investigate immune cell infiltration in DM. Our results showed that the selected autophagy-related genes significantly influenced the infiltration of multiple immune cells, such as B cells, macrophages, and natural killer cells. Finally, we assessed the diagnostic sensitivity of CCL2, CDKN1A, FOS, MYC, and TNFSF10 for DM. The results showed the area under the curve (AUC) values of the ROC were $0.855,0.889,0.744,0.826$, and 0.816 , respectively. The combined genes' diagnostic AUC value was 0.951 .

Conclusions: CCL2, CDKN1A, FOS, MYC, and TNFSF10 are potential diagnostic biomarkers for DM.

Keywords: Dermatomyositis; autophagy; biomarkers; bioinformatics analysis

Submitted Dec 07, 2021. Accepted for publication Feb 16, 2022.

doi: $10.21037 / \mathrm{atm}-22-70$

View this article at: https://dx.doi.org/10.21037/atm-22-70 


\section{Introduction}

Dermatomyositis (DM) is an autoimmune disease characterized by idiopathic inflammatory myopathy with distinguishing clinical features. For adult DM, the incidence rate is low, at about $1 / 100,000$, although the incidence rate appears to be increasing over time (1). DM can be divided into several subtypes, distinguished by different clinical outcomes with varied manifestations. A detailed physical examination is fundamental for diagnosing DM patients. In addition to the physical examination, a better understanding of the critical role played by molecular alterations in DM occurrence and progression is needed. Several gene alterations have been significantly correlated with DM, such as DNA methylation, and in particular, human leukocyte antigens (HLA), histone modification, miRNA, and major histocompatibility complex (MHC) polymorphisms. Since DM is an autoimmune disease, several autoimmune antibodies, such as myositis-specific autoantibodies (MSAs), can be used to diagnose the idiopathic inflammatory myopathies in patients (2). MSAs can be diagnostic markers and may also significantly influence the disease process. The pathogenesis of DM is still unclear, and the main underlying mechanisms may include genetic alterations, environmental factors, and immune mechanisms. There is an urgent need to identify complementary or novel molecular biomarkers to understand the pathogenesis of DM more extensively, and these may also be identified as potential therapeutic targets. DM is always accompanied by inflammation, and several recent studies have suggested that the autophagy process is significantly correlated with inflammation and the immune response (3-5).

To date, only a few studies have investigated autophagy's role in DM (6-8). Girolamo et al. revealed that the autophagy markers LC3 and p62 were more highly expressed in immune-mediated necrotizing myopathy (IMNM) than in DM or polymyositis (PM), and they mainly functioned as regulators of inflammation (8). Shu et al. showed that $\mathrm{CD}^{+} \mathrm{T}$ cells were significantly decreased in PM/DM patients' peripheral blood and that autophagy may play a protective role for these patients (7). Immunoglobulin is the most commonly used therapy regimen for treating autoimmune diseases, including DM. However, the underlying mechanism of its activation remains poorly understood. Das et al. proved that immunoglobulin mediates the anti-inflammatory effects in peripheral blood mononuclear cells by inducing autophagy (9). Day et al. showed that high mobility group box protein 1 (HMGB1) was significantly increased in IMNM and inclusion body myositis (IBM), and amongst its fundamental biological functions it also acts as an autophagy regulator (10). Furthermore, along with gene alterations, long non-coding RNAs (lncRNAs) also play key roles in $\mathrm{DM}$ and autophagy. Li et al. indicated that differentially expressed lncRNAs were detected in DM patients and played a critical role in the autophagy process (11). These results show that autophagy may play a significant role in various myositis subtypes, including DM. However, few studies have comprehensively investigated the expression levels of autophagy-related genes, their other biological functions, or their roles in immune cell infiltration in DM. Hence, we performed this study. We present the following article in accordance with the STREGA reporting checklist (available at https://atm.amegroups.com/article/ view/10.21037/atm-22-70/rc).

\section{Methods}

\section{Raw data}

We collected three datasets of dermatomyositis-related transcriptome from the Gene Expression Omnibus (GEO) database; GSE1551 contained 10 healthy samples and 13 DM samples, GSE46239 contained 4 healthy samples and 48 DM samples, and GSE143323 contained 20 healthy samples and 39 DM samples. The mRNA expression matrices of the three datasets were normalized and merged using the "sva" package in R software (https://www.r-project.org/). Finally, an mRNA expression matrix of 34 healthy samples and $100 \mathrm{DM}$ samples was obtained. The study was conducted in accordance with the Declaration of Helsinki (as revised in 2013).

\section{Analysis of differentially expressed genes (DEGs)}

The healthy and DM samples were grouped and analyzed using $\mathrm{R}$ software's "limma" package. A $\log 2$ fold change $|\log \mathrm{FCl}|>1$ and false discovery rate (FDR) $<0.05$ were used as the criteria to identify DEGs.

\section{Functional enrichment analysis of DEGs}

We performed Gene Ontology (GO) and Kyoto Encyclopedia of Genes and Genomes (KEGG) functional enrichment analyses of the DEGs using the "clusterProfiler" package in $\mathrm{R}$ software, and the results were visualized with the "ggplot2" package. 


\section{Identification of DM autophagy-related genes}

We collected the list of autophagy-related genes from the Human Autophagy Database (HADb, http://www. autophagy.lu/). The DM autophagy genes and DEGs were intersected, and the DM-related autophagy genes were identified for further analysis.

\section{The expression levels of autophagy-related genes in DM and the ROC curve of the multi-index diagnostic model}

We analyzed the expression levels of the autophagy-related genes between healthy samples and DM samples. We drew the receiver operator characteristic (ROC) curve of the expression levels of the autophagy-related genes in DM using the "pROC" package. We then used the "glm" R function to linearly combine five DM autophagy-related genes to construct a multi-index diagnostic model.

\section{Single sample gene set enrichment analysis (ssGSEA)}

We used ssGSEA to analyze the abundance of immune cells in healthy and DM patients, and 28 types of immune cells were identified (12). We compared the infiltration of immune cells in healthy and DM samples. Furthermore, we also analyzed the correlation between the abundance of immune cells and the expression levels of autophagy-related genes in DM.

\section{Statistical analysis}

All statistical analyses were completed using $\mathrm{R}$ software. The DEGs were analyzed with the "limma" package, with the threshold set to $|\log \mathrm{FC}|>1$ and FDR $<0.05$. The Wilcoxon non-parametric test was used to compare two groups, and the correlation analyses were conducted using Spearman's method. A P value $<0.05$ was considered statistically significant.

\section{Results}

Principal component analysis (PCA) of raw transcriptome data before and after data normalization: results of DEGs in $D M$

The PCA analysis indicated that, before normalization, the principal components of mRNA expressions in the GSE1511, GSE4639, and GSE143323 datasets were significantly different (Figure 1A). Nevertheless, the principal components of mRNA expressions in these three datasets were at the same level after standardization (Figure 1B).

Compared with healthy samples, 143 genes were upregulated, and 14 were downregulated in the DM samples. The heatmap shows the 100 top-ranked upregulated and downregulated genes in DM (Figure 1C).

\section{Functional enrichment analysis of the DEGs in DM}

The results of the GO functional enrichment analysis of the DEGs in DM indicated that response to virus, defense response to virus, type I interferon signaling pathway, cellular response to type I interferon, and response to type I interferon were the top five biological processes (BPs); collagen-containing extracellular matrix, blood microparticle, myofibril, platelet alpha granule lumen, and myosin filament were the top five cell components (CC); cytokine activity, chemokine receptor binding, chemokine activity, double-stranded RNA binding, and CCR chemokine receptor binding were the top five molecular functions (MFs) (Figure 2A).

The results of the KEGG analysis indicated that Coronavirus disease-COVID-19, Influenza A, Hepatitis C, Epstein-Barr virus infection, Viral protein interaction with cytokine and cytokine receptor, Chemokine signaling pathway, Measles, Complement and coagulation cascades, Toll-like receptor signaling pathway, and Pertussis were the top 10 enrichment KEGG pathways (Figure 2B).

\section{Expression levels of autophagy-related genes in DM and bealthy samples}

Five genes were obtained from the intersection of $157 \mathrm{DM}$ DEGs and 223 autophagy genes. These were chemokine (C-C motif) ligand 2 (CCL2), cyclin-dependent kinase inhibitor 1A (CDKN1A), fos proto-oncogene, AP-1 transcription factor subunit (FOS), MYC proto-oncogene, BHLH transcription factor (MYC), and TNF superfamily member 10 (TNFSF10) (Figure 3).

Compared with healthy samples, the expression levels of these five genes were higher in the DM samples (Figure 4).

\section{Accuracy of the single- and multi-gene expression levels in} the diagnosis of DM (ROC curve)

At the single gene expression level, the AUC values of the ROC curve for DM diagnosis were as follows: CCL2 
A

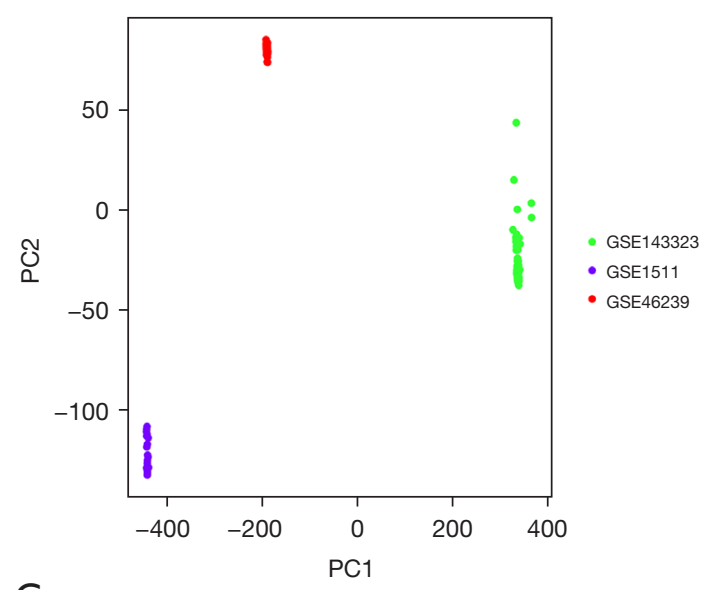

B

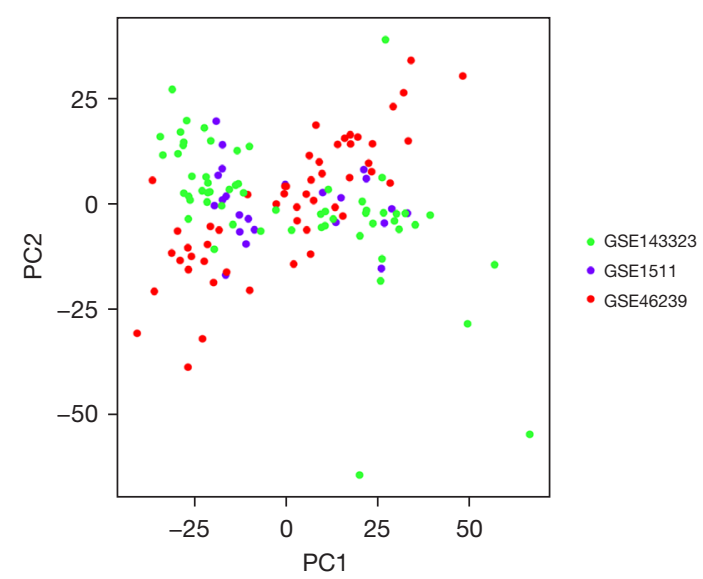

C

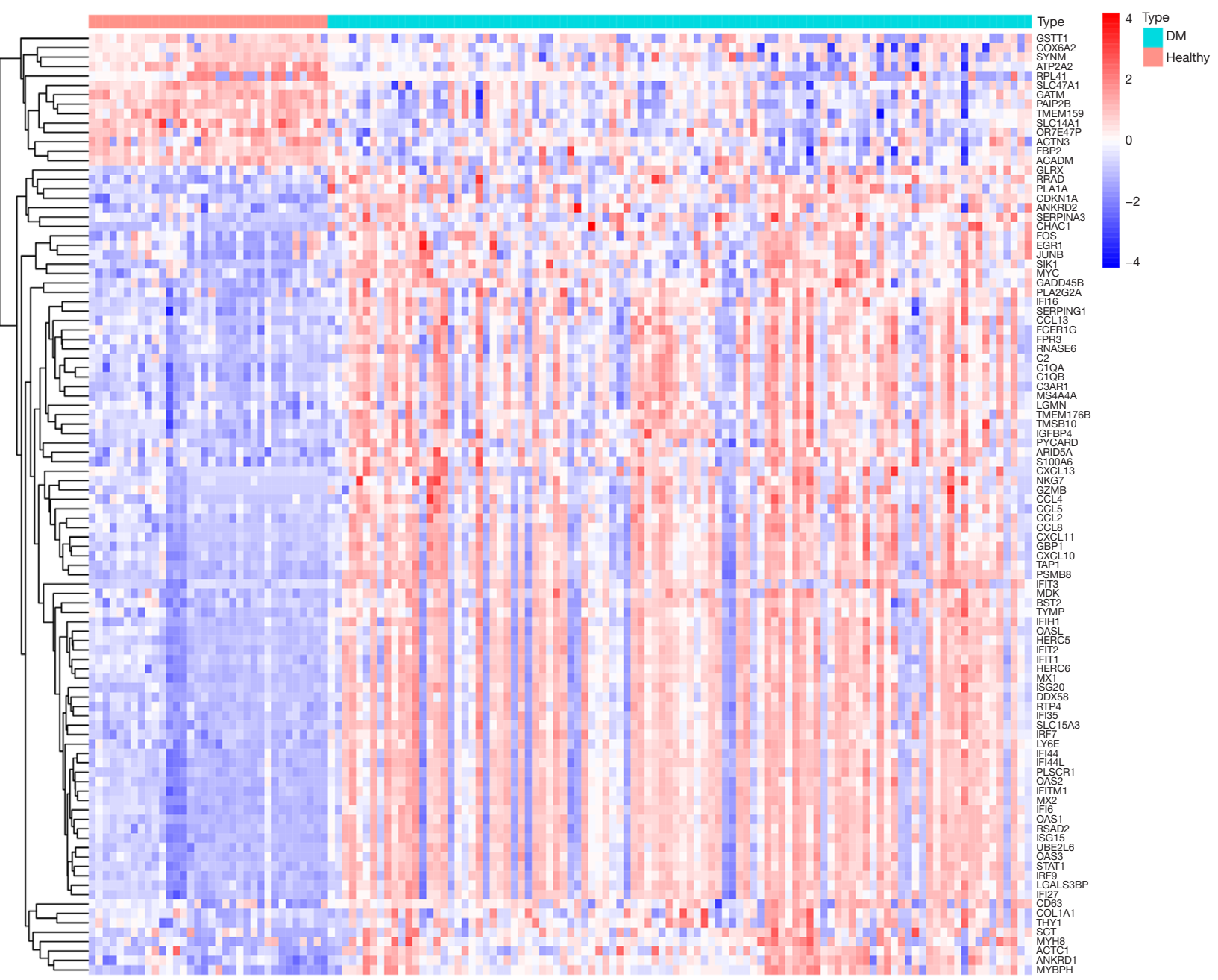

Figure 1 PCA analysis of the raw transcriptome data before and after data normalization. (A) PCA analysis before data normalization; (B) PCA analysis after data normalization. Purple represents the principal component of GSE1511, red represents the principal component of GSE46239, and green represents the principal component of GSE143323; (C) heatmap of the top 50 rank DEGs in DM. PCA, principal component analysis; DEGs, differentially expressed genes; DM, dermatomyositis. 

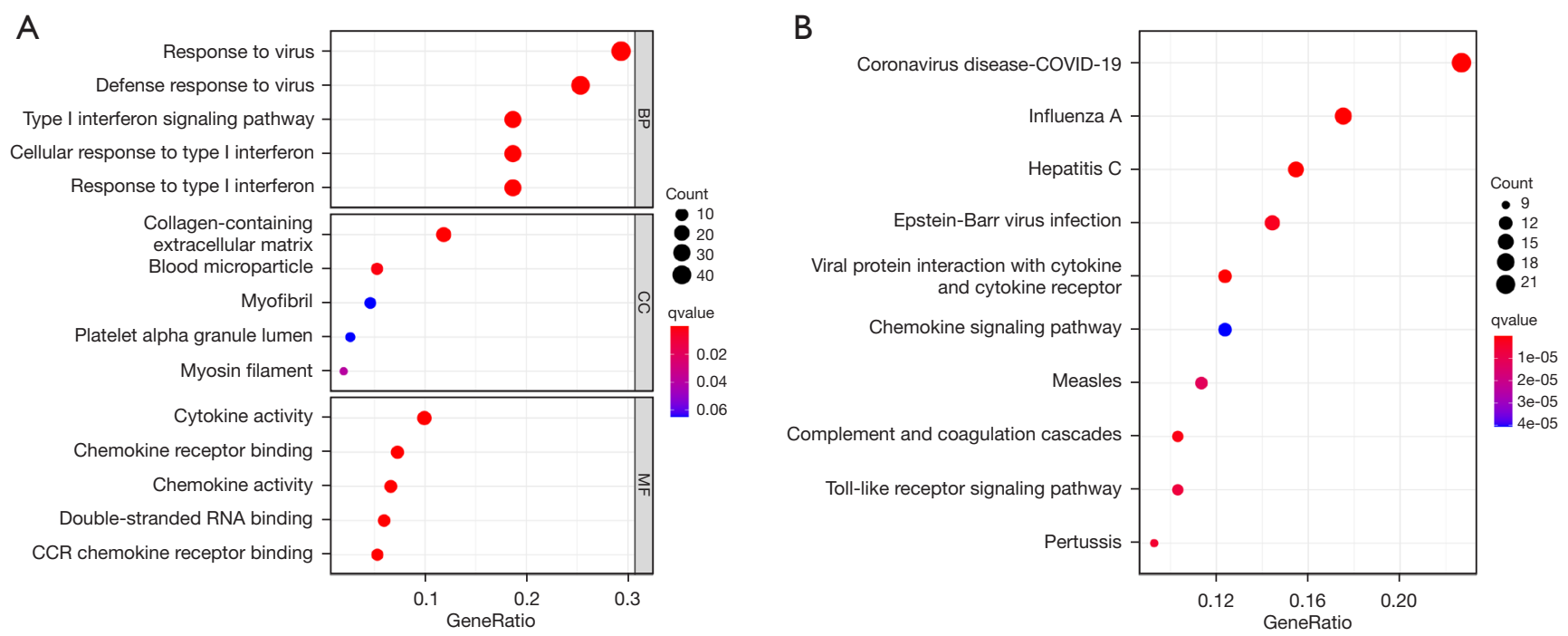

Figure 2 Functional enrichment analysis results of the DEGs in DM. (A) Bubble diagram of the GO functional enrichment analysis, including bio-logical processes, cell components, and molecular functions; (B) bubble diagram of the top 10 KEGG pathways. The bubble size represents the number of enrichment genes, and the color represents the value. DEGs, differentially expressed genes; GO, Gene Ontology; KEGG, Kyoto Encyclopedia of Genes and Genomes; DM, dermatomyositis; BP, biological process; CC, cell components; MFshid, molecular function.

A

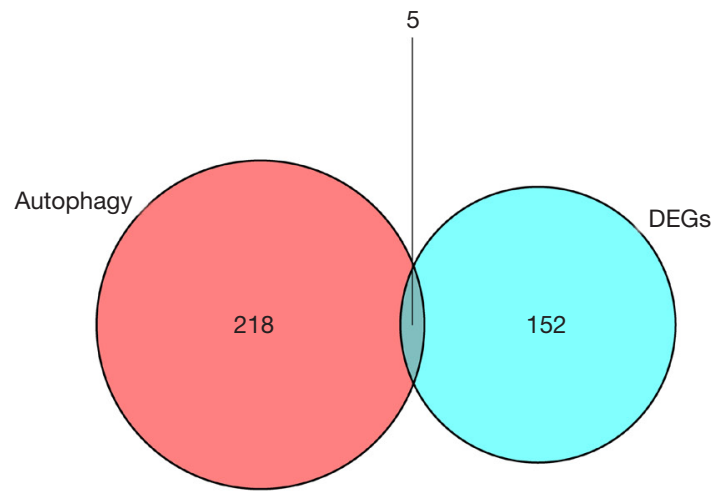

B

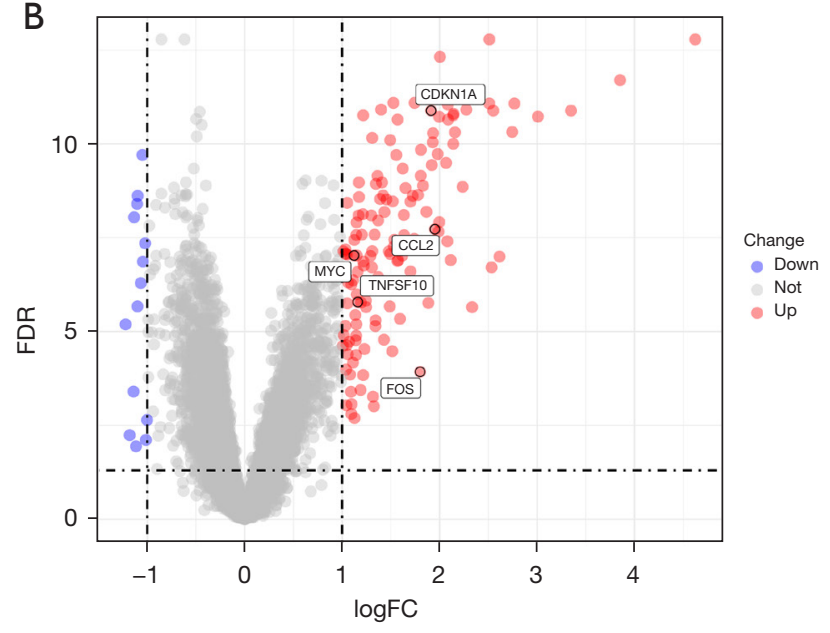

Figure 3 Intersection of DEGs and autophagy genes. (A) Venn diagram of DM DEGs and autophagy genes; (B) volcano map of the specific locations of intersecting genes in all genes. DEGs, differentially expressed genes; DM, dermatomyositis; FDR, false discovery rate.

$=0.855, C D K N 1 A=0.889, F O S=0.744, M Y C=0.826$, and TNFSF10 $=0.816$ (Figure 5A-5E).

After linear fitting of the multi-gene expression model $($ model $=-19.978+0.769 \times C C L 2+1.226 \times C D K N 1 A$ $+0.487 \times F O S+0.235 \times M Y C+0.460 \times T N F S F 10)$, the AUC value of the ROC curve of the multi-gene combined diagnosis of DM was 0.951 (Figure $5 F$ ).

\section{Correlation analysis of immune cell infiltration levels and the expression levels of five DM autophagy-related genes.}

Compared with healthy samples, 21 out of 28 immune cells showed high infiltration in the DM samples, including activated CD8 T cells, central memory CD8 T cells, and effector memory CD8 T cells (Figure 6A). 
A

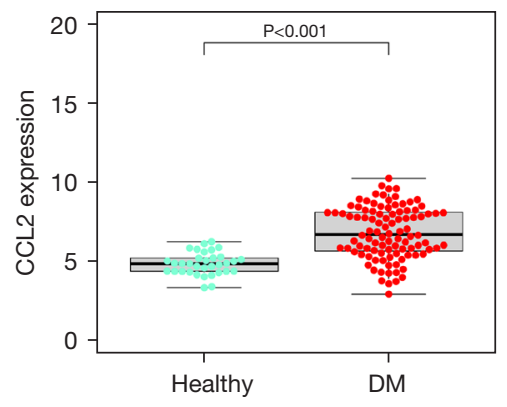

D

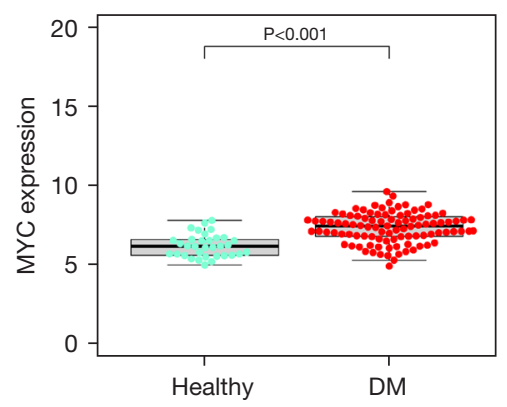

B

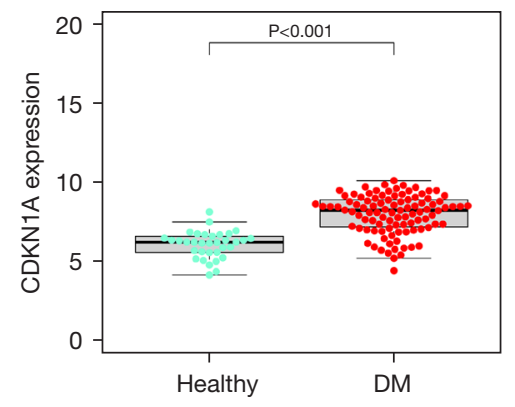

$\mathrm{E}$

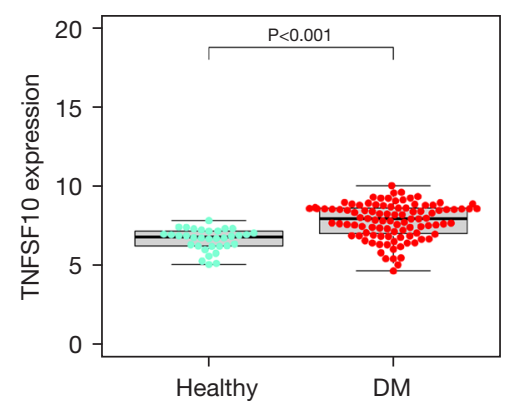

C

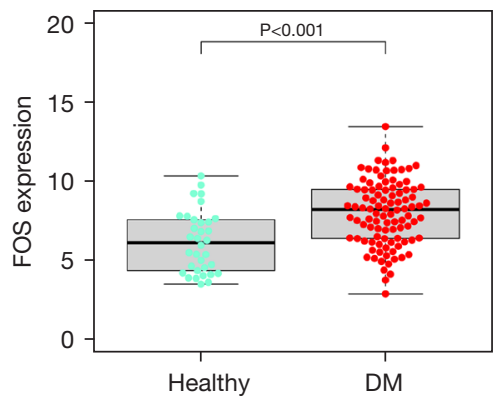

Figure 4 Expression levels of autophagy-related genes in DM and healthy samples. (A) CCL2; (B) CDKN1A; (C) FOS; (D) MYC; (E) TNFSF10. DM, dermatomyositis; CCL2, C-C motif chemokine ligand 2; CDKN1A, cyclin-dependent kinase inhibitor 1A; FOS, Fos proto-oncogene, AP-1 transcription factor subunit; MYC, MYC proto-oncogene, BHLH transcription factor; TNFSF10, TNF superfamily member 10.

The results of the correlation analysis showed a positive correlation between the expression level of five DM autophagy-related genes and the abundance of immune cell infiltration, especially CCL2 and TNFSF10 (Figure 6B).

\section{Discussion}

DM and PM are known as inflammatory myopathies and are amongst the most common autoimmune diseases. Several studies have reported on the inflammatory process and, in particular, the role of the cells $(13,14)$. Autophagy is the process whereby subcellular components are degraded through the lysosomal signaling pathway (15). Autophagy has multiple functions in addition to the immune response and can act as the balancing factor for lymphocytes by eliminating intracellular pathogens and antigens to inhibit cell death. We know that DM is characterized by antigen presentation, immune system dysfunction, and muscle cell death and that autophagy is associated with many of these factors. However, the roles that autophagy-related genes play in DM require further exploration. Thus, we performed this study to learn whether autophagy-related genes were differentially expressed in DM than in healthy individuals. Additionally, we aimed to understand the main biological functions of these genes to identify them as complementary or novel biomarkers of the disease. By increasing our knowledge of the underlying mechanisms of autophagy-related genes in DM, there is the potential that they can assist in the diagnosis, assessment, and even treatment of DM.

The present study showed that 143 genes were upregulated and 14 were downregulated in DM samples compared with healthy samples. To understand the functions of these DEGs between the DM and healthy control groups, we conducted a functional enrichment analysis that revealed these DEGs play significant roles in various functions, including the type I interferon signaling pathway, cytokine activity, chemokine activity, CCR chemokine receptor binding, double-stranded RNA binding, and blood microparticles. Several previous studies have confirmed our finding that the type I interferon signaling pathway plays a critical role in DM (16-20). Baechler et al. revealed that increased expression levels of type I interferon signatures are associated with DM 
A
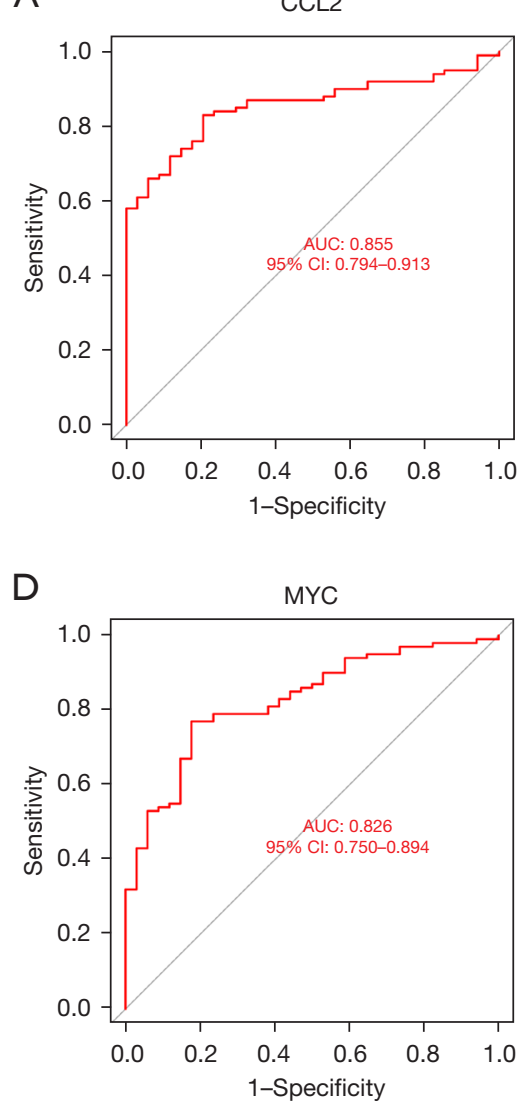

B

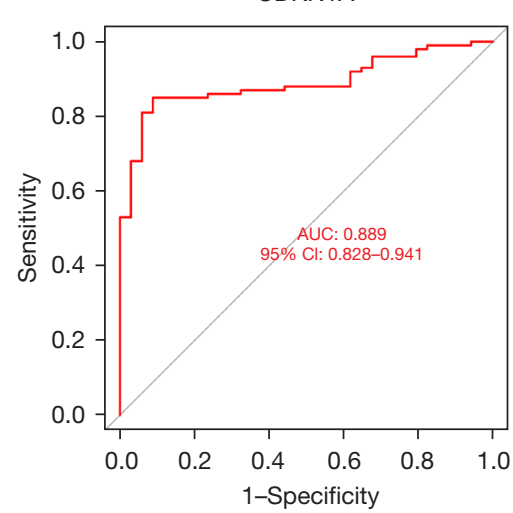

E

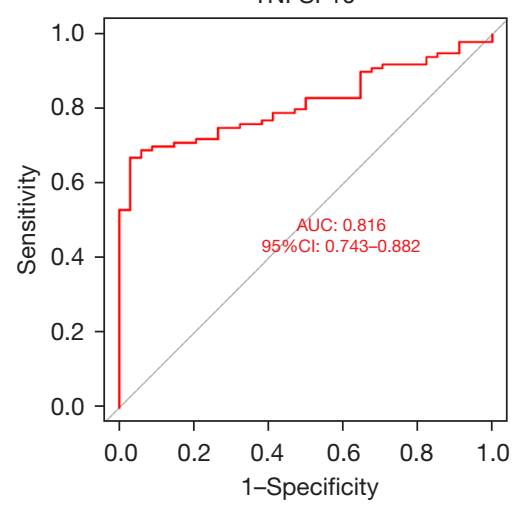

C

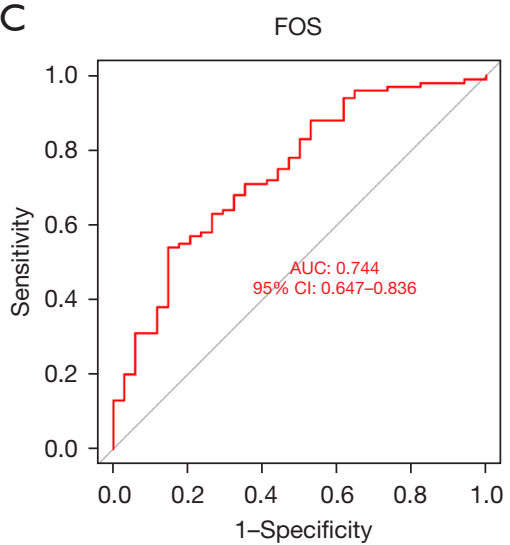

$\mathrm{F}$

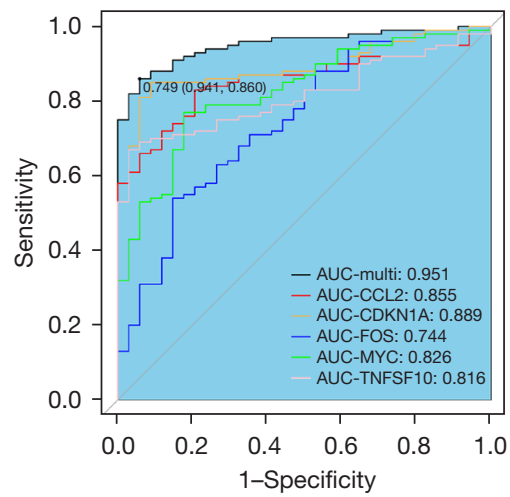

Figure 5 Accuracy of the single- and multi-gene expression levels in the diagnosis of DM (ROC curve). (A) CCL2; (B) CDKN1A; (C) FOS; (D) MYC; (E) TNFSF10; (F) multi-index combined diagnosis. CCL2, C-C motif chemokine ligand 2; CDKN1A, cyclin-dependent kinase inhibitor 1A; FOS, Fos proto-oncogene, AP-1 transcription factor subunit; MYC, MYC proto-oncogene, BHLH transcription factor; TNFSF10, TNF superfamily member 10; ROC, receiver operator characteristic; DM, dermatomyositis.

activity (16), and Gitiaux et al. reported type I interferon changes in juvenile DM (17). The type I interferon signature was also found to be highly expressed in the MDA5 $^{+}$DM subtype (19). Based on these findings of type I interferon signaling pathway changes as critical events in DM pathogenesis, researchers have explored the efficacy of type I interferon inhibitors in the clinical treatment of DM. Ladislau et al. showed that JAK inhibitors improved symptoms and reduced type I interferon levels in DM patients (18). In addition to the interferon signaling pathway, other cytokines may have individual or multiple influences on DM (21). Moneta et al. showed that tumor necrosis factor (TNF) expression was significantly increased in DM patients prior to glucocorticoid therapy (22). Interleukin-6 (IL-6), interleukin-17A $(I L-17 A)$, and interleukin-15 $(I L-15)$ have also been implicated in DM progression (23-25). IL-15 has been identified as a crucial biomarker for predicting the development of rapidly progressive interstitial lung disease in DM/PM patients (25). These findings show that cytokine and type I interferon signaling pathway changes are key events in the onset and progression of DM.

Given the critical role of autophagy in DM and its regulation by autophagy-related genes, we performed an intersection between DEGs and autophagy-related genes and selected the intersected genes for further analysis. The results showed that CCL2, CDKN1A, FOS, MYC, and TNFSF10 were implicated. All exhibited significantly higher expressions in DM patients than healthy samples. Unfortunately, few studies have investigated the role of these genes in DM. Oda et al. found that DM patients with interstitial pneumonia (IP) who died had significantly higher 
A 宁 DM 户 Healthy

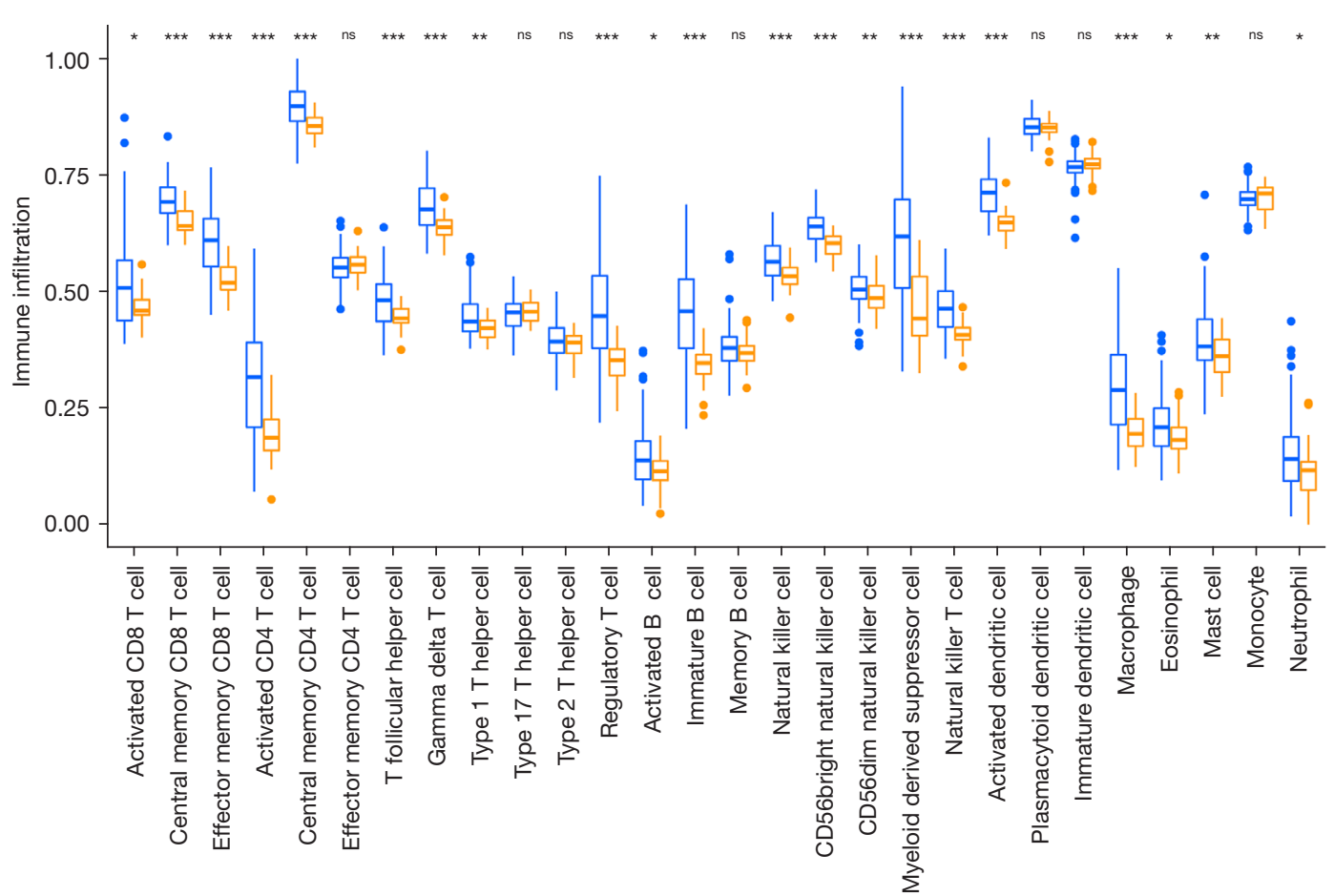

B

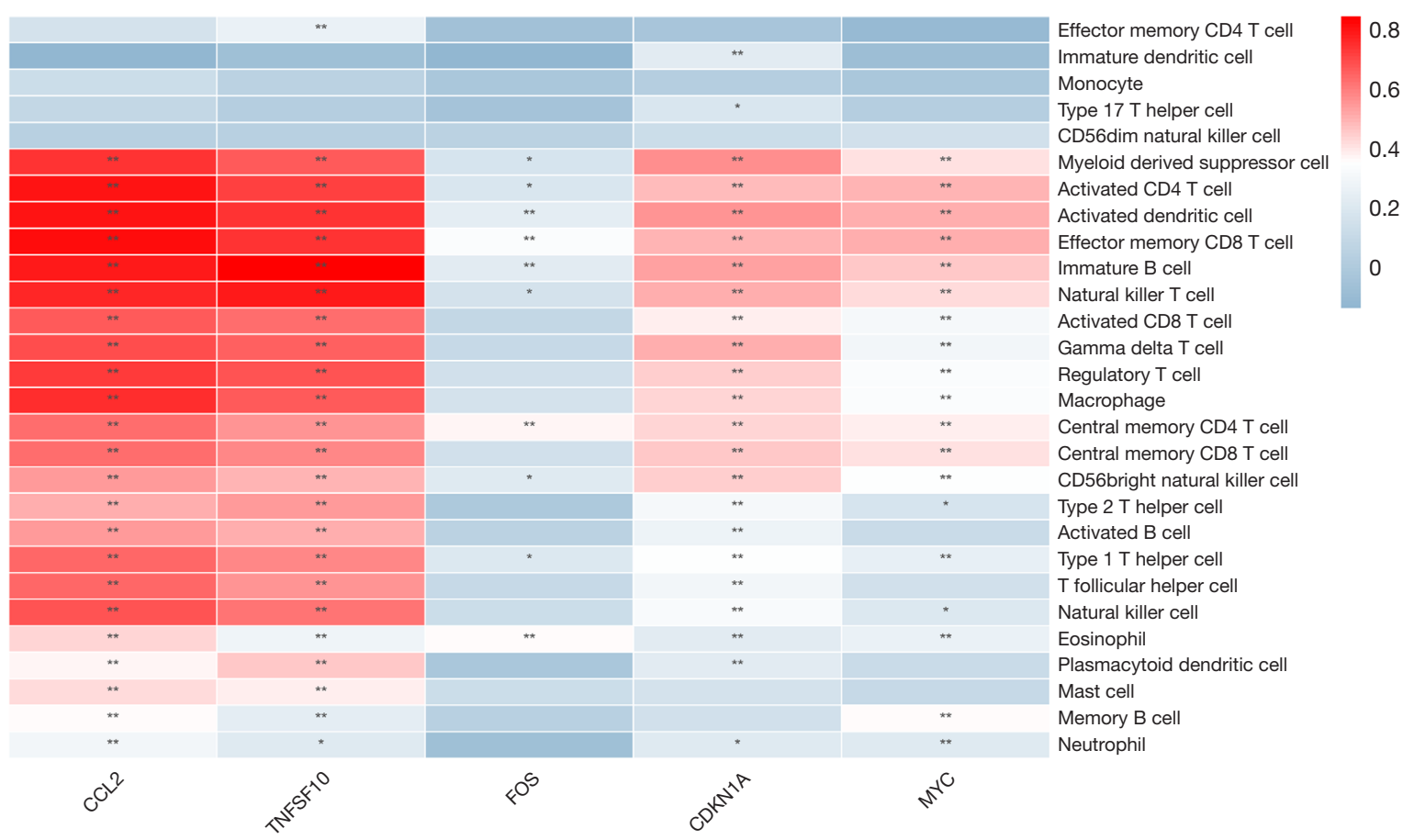

Figure 6 Correlation analysis of immune cell infiltration and the expression levels of five DM autophagy-related genes. (A) Boxplot of differential analysis of immune cell infiltration levels in healthy and DM samples; (B) heatmap of immune cell infiltration levels and DM autophagy-related genes, where red represents a strong positive correlation, and gray represents a weak positive correlation. ${ }^{\text {ns }} \mathrm{P}>0.05$, ${ }^{*} \mathrm{P}<0.05,{ }^{* *} \mathrm{P}<0.01,{ }^{* * *} \mathrm{P}<0.001$. DM, dermatomyositis. 
CCL2 serum levels than those who survived (26). This result indicates that CCL2 is not only significantly associated with DM occurrence but also with its progression. CCL2 has also been implicated in systemic sclerosis (27). To date, CDKN1A/p21 has not been investigated in DM. Seleznik et al. reported that $C D K N 1 A$ promotes inflammation in autoimmune pancreatitis (28). Aljabban et al. indicated that $M Y C$ was associated with interferon signaling pathway changes and was upregulated in DM patients (29). TNFSF10 is a critical inflammation regulator and has been shown to play a key role in several diseases associated with inflammatory changes, including various cancers and Alzheimer's disease (30-32). The above results confirm that autophagy plays a significant role in DM and other inflammatory diseases.

The functional enrichment analysis and the intersection of autophagy-related genes showed that their biological function was enriched in the inflammation and cytokine signaling pathways. Given that immune cells play an essential role in the inflammation process, we were curious to investigate immune cell infiltration in DM patients. Our results showed that the final selection of autophagyrelated genes significantly influenced the infiltration of multiple immune cells. Immune system changes are the primary underlying factor in autoimmune diseases, and immune cell dysregulation has been observed in various autoimmune diseases, such as systemic lupus erythematosus (SLE), Behçet's disease, and MPO-ANCA vasculitis (33-35). However, few studies have clarified the role of immune cells in DM. Cassius et al. reported T cell dysregulation in patients with active DM (36), and B cells, macrophages, and natural killer cells have also been shown to significantly affect DM progression (37-39). This study identified CCL2, CDKN1A, FOS, MYC, and TNFSF10 as potential biomarkers for DM. Shu et al found that autophagy may serve a potential protective role in the peripheral blood T cells of patients with DM (7). Our results show that activated CD8 $\mathrm{T}$ cells, central memory CD8 $\mathrm{T}$ cells and effector memory CD8 $\mathrm{T}$ cells were high infiltration in the DM samples, therefore, CCL2, CDKN1A, FOS, MYC, and TNFSF10 of hub autophagy genes may treat DM by regulating immune cells. However, the limitation of our study is the lack of expression and functional investigation of hub autophagy genes.

\section{Conclusions}

The autophagy-related genes CCL2, CDKN1A, FOS, MYC, and TNFSF10 showed significantly higher expressions in DM samples than healthy samples, and their most important biological functions include cytokine and type I interferon signaling pathway regulations. Additionally, they have a significant influence on immune cell infiltration in DM. They may also serve as potential biomarkers for diagnosing DM.

\section{Acknowledgments}

Funding: None.

\section{Footnote}

Reporting Checklist: The authors have completed the STREGA reporting checklist. Available at https://atm. amegroups.com/article/view/10.21037/atm-22-70/rc

Conflicts of Interest: All authors have completed the ICMJE uniform disclosure form (available at https://atm. amegroups.com/article/view/10.21037/atm-22-70/coif). The authors have no conflicts of interest to declare.

Ethical Statement: The authors are accountable for all aspects of the work in ensuring that questions related to the accuracy or integrity of any part of the work are appropriately investigated and resolved. The study was conducted in accordance with the Declaration of Helsinki (as revised in 2013).

Open Access Statement: This is an Open Access article distributed in accordance with the Creative Commons Attribution-NonCommercial-NoDerivs 4.0 International License (CC BY-NC-ND 4.0), which permits the noncommercial replication and distribution of the article with the strict proviso that no changes or edits are made and the original work is properly cited (including links to both the formal publication through the relevant DOI and the license). See: https://creativecommons.org/licenses/by-nc-nd/4.0/.

\section{References}

1. Bendewald $M$, Wetter D, Li X, et al. Incidence of dermatomyositis and clinically amyopathic dermatomyositis: a population-based study in Olmsted County, Minnesota. Archives of dermatology 2010;146:26-30.

2. Wolstencroft P, Fiorentino D. Dermatomyositis Clinical 
and Pathological Phenotypes Associated with MyositisSpecific Autoantibodies. Current rheumatology reports 2018;20:28.

3. Bergveld P, Wiersma J, Meertens H. Extracellular potential recordings by means of a field effect transitor without gate metal, called OSFET. IEEE transactions on bio-medical engineering 1976;23:136-44.

4. Zhong Z, Sanchez-Lopez E, Karin M. Autophagy, Inflammation, and Immunity: A Troika Governing Cancer and Its Treatment. Cell 2016;166:288-98.

5. Matsuzawa-Ishimoto Y, Hwang S, Cadwell K. Autophagy and Inflammation. Annual review of immunology 2018;36:73-101.

6. Cappelletti C, Galbardi B, Kapetis D, et al. Autophagy, inflammation and innate immunity in inflammatory myopathies. PloS one 2014;9:e111490.

7. Shu X, Chen F, Peng Q, et al. Potential role of autophagy in T-cell survival in polymyositis and dermatomyositis. Molecular medicine reports 2017;16:1180-8.

8. Girolamo F, Lia A, Annese T, et al. Autophagy markers LC3 and p62 accumulate in immune-mediated necrotizing myopathy. Muscle \& nerve 2019;60:315-27.

9. Das M, Karnam A, Stephen-Victor E, et al. Intravenous immunoglobulin mediates anti-inflammatory effects in peripheral blood mononuclear cells by inducing autophagy. Cell death \& disease 2020;11:50.

10. Day J, Otto S, Cash K, et al. Aberrant Expression of High Mobility Group Box Protein 1 in the Idiopathic Inflammatory Myopathies. Frontiers in cell and developmental biology 2020;8:226.

11. Li L, Zuo X, Liu D, et al. Plasma exosomal RNAs has potential as both clinical biomarkers and therapeutic targets of dermatomyositis. Rheumatology (Oxford, England) 2021.

12. Charoentong P, Finotello F, Angelova M, et al. Pancancer Immunogenomic Analyses Reveal GenotypeImmunophenotype Relationships and Predictors of Response to Checkpoint Blockade. Cell reports 2017;18:248-62.

13. Zhu M, Deng G, Xing C, et al. BECN2 (beclin 2)-mediated non-canonical autophagy in innate immune signaling and tumor development. Autophagy 2020;16:2310-2.

14. Riffelmacher T, Richter FC, Simon AK. Autophagy dictates metabolism and differentiation of inflammatory immune cells. Autophagy 2018;14:199-206.

15. Choi A, Ryter S, Levine B. Autophagy in human health and disease. The New England journal of medicine 2013;368:651-62.
16. Baechler E, Bilgic H, Reed A. Type I interferon pathway in adult and juvenile dermatomyositis. Arthritis research \& therapy 2011;13:249.

17. Gitiaux C, Latroche C, Weiss-Gayet M, et al. Myogenic Progenitor Cells Exhibit Type I Interferon-Driven Proangiogenic Properties and Molecular Signature During Juvenile Dermatomyositis. Arthritis \& rheumatology (Hoboken, NJ) 2018;70:134-45.

18. Ladislau L, Suárez-Calvet X, Toquet S, et al. JAK inhibitor improves type I interferon induced damage: proof of concept in dermatomyositis. Brain : a journal of neurology 2018;141:1609-21.

19. Cassius C, Amode R, Delord M, et al. MDA5 Dermatomyositis Is Associated with Stronger Skin Type I Interferon Transcriptomic Signature with Upregulation of IFN- $\kappa$ Transcript. The Journal of investigative dermatology 2020;140:1276-9.e7.

20. Kuriyama Y, Shimizu A, Kanai S, et al. The synchronized gene expression of retrotransposons and type I interferon in dermatomyositis. Journal of the American Academy of Dermatology 2021;84:1103-5.

21. Arshanapalli A, Shah M, Veerula V, et al. The role of type I interferons and other cytokines in dermatomyositis. Cytokine 2015;73:319-25.

22. Moneta G, Pires Marafon D, Marasco E, et al. Muscle Expression of Type I and Type II Interferons Is Increased in Juvenile Dermatomyositis and Related to Clinical and Histologic Features. Arthritis \& rheumatology (Hoboken, NJ) 2019;71:1011-21.

23. Yang M, Cen X, Xie Q, et al. Serum interleukin-6 expression level and its clinical significance in patients with dermatomyositis. Clinical \& developmental immunology 2013;2013:717808.

24. Silva M, Oba-Shinjo S, Marie S, et al. Serum interleukin$17 \mathrm{~A}$ level is associated with disease activity of adult patients with dermatomyositis and polymyositis. Clinical and experimental rheumatology 2019;37:656-62 .

25. Shimizu T, Koga T, Furukawa K, et al. IL-15 is a biomarker involved in the development of rapidly progressive interstitial lung disease complicated with polymyositis/dermatomyositis. Journal of internal medicine 2021;289:206-20.

26. Oda K, Kotani T, Takeuchi T, et al. Chemokine profiles of interstitial pneumonia in patients with dermatomyositis: a case control study. Scientific reports 2017;7:1635.

27. Assassi S, Mayes M. What does global gene expression profiling tell us about the pathogenesis of systemic sclerosis? Current opinion in rheumatology 
2013;25:686-91.

28. Seleznik G, Reding T, Peter L, et al. Development of autoimmune pancreatitis is independent of CDKN1A/p21mediated pancreatic inflammation. Gut 2018;67:1663-73.

29. Aljabban J, Syed S, Syed S, et al. Investigating genetic drivers of dermatomyositis pathogenesis using metaanalysis. Heliyon 2020;6:e04866.

30. Cantarella G, Di Benedetto G, Puzzo D, et al. Neutralization of TNFSF10 ameliorates functional outcome in a murine model of Alzheimer's disease. Brain : a journal of neurology 2015;138:203-16.

31. Cullen S, Martin S. Fas and TRAIL 'death receptors' as initiators of inflammation: Implications for cancer. Seminars in cell \& developmental biology 2015;39:26-34.

32. Huang B, Yu H, Li Y, et al. Upregulation of long noncoding TNFSF10 contributes to osteoarthritis progression through the miR-376-3p/FGFR1 axis. Journal of cellular biochemistry 2019;120:19610-20.

33. Hamzaoui K. Th17 cells in Behçet's disease: a new immunoregulatory axis. Clinical and experimental rheumatology 2011;29:S71-6.

34. Herrada A, Escobedo N, Iruretagoyena M, et al. Innate Immune Cells' Contribution to Systemic Lupus Erythematosus. Frontiers in immunology 2019;10:772.

Cite this article as: Wang L, Fang D, Liu Y. Autophagyrelated genes are potential diagnostic biomarkers for dermatomyositis. Ann Transl Med 2022;10(4):228. doi: 10.21037/atm-22-70
35. Free M, Stember K, Hess J, et al. Restricted myeloperoxidase epitopes drive the adaptive immune response in MPO-ANCA vasculitis. Journal of autoimmunity 2020;106:102306.

36. Cassius C, Branchtein M, Battistella M, et al. Persistent deficiency of mucosal-associated invariant $T$ cells during dermatomyositis. Rheumatology (Oxford, England) 2020;59:2282-6.

37. Gonzalez-Amaro R, Alcocer-Varela J, Alarcón-Segovia D. Natural killer cell activity in dermatomyositispolymyositis. The Journal of rheumatology 1987;14:307-10.

38. Piper C, Wilkinson M, Deakin C, et al. CD19CD24CD38 B Cells Are Expanded in Juvenile Dermatomyositis and Exhibit a Pro-Inflammatory Phenotype After Activation Through Toll-Like Receptor 7 and Interferon- $\alpha$. Frontiers in immunology 2018;9:1372.

39. Jiang T, Huang Y, Liu H, et al. Reduced miR-146a Promotes REG3A Expression and Macrophage Migration in Polymyositis and Dermatomyositis. Frontiers in immunology 2020;11:37.

(English Language Editor: D. Fitzgerald) 